\title{
INFRARED EMISSION LINES FROM CELESTIAL SOURCES
}

\author{
by Robert J. GootD \\ (University of California, San Diego La Jolla, California, U. S. A.)
}

RÉSUMt. - On discute la probabilité d'observer des raies d'émission intenses dans l'infrarouge. La raie 12,8 $\mu$ qui est produite par une transition entre les composantes fines de l'état fondamental de l'ion $\mathrm{Ne}^{+}$pourrait être émise par les régions H II galactiques et, peut-être, par des enveloppes stellaires étendues. La raie $28 \mu$ émise par une transition de rotation $J=2, J=0$ de la molécule de (para) hydrogène pourrait être observée dans les régions $H I$ interstellaires et par des protoétoiles en voie de contraction. On donne une estimation de l'intensité de ces raies d'émission que l'on peut s'attendre à observer.

ABstRaCT. - Some possible strong astronomical emission lines in the infrared region are discussed. The line at 12.8 microns, which results from a transition between the fine structure components of the ground state of the ion $\mathrm{Ne}^{+}$, is thought to be emitted from galactic $\mathrm{H}$ II regions and also possibly from extended stellar envelopes. The line at 28 microns is emitted in the $J=2$ to $J=0$ rotational transition in the (para) hydrogen molecule, and could be emitted from interstellar $H I$ regions and from collapsing protostars. Estimates of the expected intensities of these astronomical emission lines are given.

l'езюме. - Обсуждена вероятность наблюдения интенсивньх эмиссионных линий в инфракрасной области. Линия $12,8 \mu$, порожденная переходом между тонкими составляющими основного состояния иона $\mathrm{Ne}^{+}$, может быть излученной галактическими областями $\mathrm{H}$ II и, быть-можіет, протяженными звездными оболочками. Линия $28 \mu$, излученная ротационным переходом $\mathbf{J}=2$ $\mathbf{J}=0$ молекулы параводорода, можкет быть наблюденной в межзвевдньх областях Н I и в протозвездах в состоянии сжатия. Дана оценка интенсивности этих эмиссионных линий, наблюдение которых можно ожидать.

Recent advances in the construction of infrared detectors lead one to consider the possible source of infrared radiation in the Galaxy. In this paper I shall attempt to make estimates of the expected fluxes from some infrared lines which seem to have the best chances of detection.

\section{Emission Lines from galactic H II Regions.}

A recent theoretical study of processes in H II regions (BURBIDGh, Gould and PotTasch 1963, hereafter referred to as " BGP ") indicated that an appreciable fraction of the energy radiated from these regions is emitted in the form of infrared lines. These lines result from transitions between the fine structure components of the ground state terms of a number of abundant ions. The separation of these levels is $\sim 0.01-0.1 \mathrm{eV}$ and the excitation is by inelastic collisions of electrons which have thermal energies of $\sim 1 \mathrm{eV}$. The lines resulting from some of the more abundant ions are listed in Table I (taken from GouLd 1963). The transitions listed are of the "forbidden" magnetic dipole type corresponding to $\Delta \mathrm{J}= \pm 1$; quadrupole transitions with $\Delta J= \pm 2$ are much weaker. Thus, for example, the $J=2$ level in $\mathrm{N}^{+}$would depopulate predominantly by $\mathrm{J}=\mathbf{2} \rightarrow 1 \rightarrow 0$, emitting two infrared photons.

Of the ions listed in Table $\mathrm{I}, \mathrm{Ne}^{+}$is probably the most abundant in $\mathrm{H}$ II regions, since neon is singly ionized in regions excited by stars of essentially the same surface temperature range that produce most of the ionization of the interstellar gas (see BGP). The amount of energy radiated per $\mathrm{cm}^{3}$ per second from an ionized region of density $n_{e}$ in the $\mathrm{Ne}^{+}$line at $12.8 \mu$ is $n_{e}^{2} \varepsilon\left(\mathrm{Ne}^{+}\right)$, where, if neon is singly ionized,

$$
\varepsilon\left(\mathrm{Ne}^{+}\right) \approx 2 \times 10^{-24} \mathrm{erg}-\mathrm{cm}^{3} / \mathrm{s} .
$$

As shown by BGP, this infrared line is one of the principal scurces of cooling in H II regions; by comparison, the intensity of the $\mathrm{H} \alpha$ line is only about one-third as large as that of the $12.8 \mu$ line. It is of interest to consider the expected $12.8 \mu$ flux from galactic H II regions, especially since this line lies within the 8-14 $\mu$ window of the atmosphere and could thus be observed from ground based instruments.

Take the following model for the galactic gas : 


\section{TABLE I}

INFRARED EMISSION LINES

\begin{tabular}{|c|c|c|c|c|}
\hline Ion & $\begin{array}{c}\text { GROUND } \\
\text { State }\end{array}$ & $\begin{array}{l}\text { Transition } \\
\left(J_{1}-J_{2}\right)\end{array}$ & $\begin{array}{c}\nu \\
\left(\mathrm{cm}^{-1}\right)\end{array}$ & $\begin{array}{c}\lambda \\
(\mu)\end{array}$ \\
\hline - & - & - & - & - \\
\hline $\mathrm{C}^{+}$ & $2 p{ }^{2} \mathbf{P}_{1 / 2}$ & $\frac{1}{2}-\frac{3}{2}$ & 64.0 & 156.3 \\
\hline $\mathrm{N}^{+}$ & $2 p^{2} \mathbf{3} \mathrm{P}_{0}$ & $\begin{array}{l}0-1 \\
1-2\end{array}$ & $\begin{array}{l}49.1 \\
82.2\end{array}$ & $\begin{array}{l}203.6 \\
121.7\end{array}$ \\
\hline$N^{+2}$ & $2 p^{2} \mathrm{P}_{1 / 2}$ & $\frac{1}{2}-\frac{3}{2}$ & 174.5 & 57.31 \\
\hline $\mathrm{O}^{+2}$ & $2 p^{2}{ }^{3} \mathrm{P}_{0}$ & $\begin{array}{l}0-1 \\
1-2\end{array}$ & $\begin{array}{l}113.4 \\
193.4\end{array}$ & $\begin{array}{l}88.18 \\
51.71\end{array}$ \\
\hline $\mathrm{O}^{+3}$ & $2 p{ }^{2} \mathrm{P}_{1 / 2}$ & $\frac{1}{2}-\frac{3}{2}$ & 386.5 & 25.87 \\
\hline $\mathrm{Ne}^{+}$ & $2 p^{5}{ }^{2} \mathrm{P}_{\mathrm{g} / 2}$ & $\frac{3}{2}-\frac{1}{2}$ & 782 & 12.79 \\
\hline $\mathrm{Ne}^{+2}$ & $2 p^{4}{ }^{3} \mathrm{P}_{2}$ & $\begin{array}{l}2-1 \\
1-0\end{array}$ & $\begin{array}{l}650 \\
277\end{array}$ & $\begin{array}{l}15.38 \\
36.10\end{array}$ \\
\hline $\mathrm{Ne}^{+4}$ & $2 p^{2} \mathrm{~s}_{0}$ & $\begin{array}{l}0-1 \\
1-2\end{array}$ & $\begin{array}{l}414 \\
698\end{array}$ & $\begin{array}{l}24.15 \\
14.33\end{array}$ \\
\hline $\mathrm{Mg}^{+3}$ & $2 p^{5}{ }^{2} \mathrm{P}_{3 / 2}$ & $\frac{3}{2}-\frac{1}{2}$ & 2226 & 4.492 \\
\hline $\mathrm{Si}^{+}$ & $3 p^{2} \mathrm{P}_{\frac{1}{2}}$ & $\frac{1}{2}-\frac{3}{2}$ & 287 & 34.8 \\
\hline$S^{+2}$ & $3 p^{2}{ }^{3} \mathrm{P}_{0}^{2}$ & $\begin{array}{l}0-1 \\
1-2\end{array}$ & $\begin{array}{l}297.2 \\
535.3\end{array}$ & $\begin{array}{l}33.65 \\
18.681\end{array}$ \\
\hline$S^{+3}$ & $3 p{ }^{2} \mathrm{P}_{\frac{1}{2}}$ & $\frac{1}{2}-\frac{3}{2}$ & 950 & 10.53 \\
\hline
\end{tabular}

the gas exists in discrete " clouds" which fill a fraction $f_{0} \sim 0.1$ of the volume $V_{d}$ of a disk of radius $r_{d} \simeq 10 \mathrm{kpc}$ and thickness $t \simeq 200 \mathrm{pc}$ containing the gas clouds

$$
\left(\mathrm{V}_{d}=\pi r_{d}^{2} t \sim 2 \times 10^{66} \mathrm{~cm}^{3}\right) ;
$$

a fraction $f_{i} \sim 0.1$ of the clouds are ionized and have a density $\left(n_{e}\right)_{0} \sim 10 \mathrm{~cm}^{-3}$. The galactic disk luminosity of the $12.8 \mu$ line from $\mathrm{Ne}^{+}$is then

(1) $\mathrm{L}_{d}\left(\mathrm{Ne}^{+}\right)=\left[n_{e}^{2} \varepsilon\left(\mathrm{Ne}^{+}\right)\right]_{c} f_{c} f_{i} \mathrm{~V}_{d}$

$$
\sim 4 \times 10^{42} \mathrm{erg} / \mathrm{s} .
$$

This figure represents an appreciable fraction of the total energy emitted beyond the Lyman limit by hot stars in the galactic disk. Clearly the line is well above the infrared stellar continuum. Even for poor resolution $\Delta \lambda / \lambda \sim 1$ the line intensity is about 4 orders of magnitude above the stellar continuum and about 3 orders of magnitude above the $e-p$ bremsstrahlung continuum from the same H II regions. To get an idea of the energy flux to be expected from the galactic disk, suppose that this source were placed at a distance $r_{d}$; then

(2) $\mathrm{F}_{a}\left(\mathrm{Ne}^{+}\right) \sim \mathrm{L}_{d}\left(\mathrm{Ne}^{+}\right) / 4 \pi r_{d}^{2}$ $\sim 4 \times 10^{-11} \mathrm{watt} / \mathrm{cm}^{2}$.

Since the noise equivalent power (essentially the minimum detectable signal) of photoconductive detectors for this wavelength region is about $2 \times 10^{-12}$ watt and may be lowered to $10^{-14}$ watt in the near future it would appear that the flux (2) should be easily detectable. Self absorption of the $12.8 \mu$ line is negligible and extinction by the interstellar grains would amount to only about $1 \mathrm{Mag} / 10 \mathrm{kpc}$ in the galactic plane. Thus, one should be able to "see" essentially the whole galaxy by means of this line. In fact, if good spectral resolution could be attained, so that the Doppler shift due to differential galactic rotation could be measured, a plot of galactic H II regions could be made as is done in radio astronomy with the $21 \mathrm{~cm}$ line.

Concerning possible discrete sources of $12.8 \mu$ emission, the Orion Nebula with an $H$ II region about $1^{\circ}$ in diameter would appear to be the strongest. The flux can be estimated from the observed $\mathrm{H}_{\alpha}$ flux (which is essentially a measure of $\int n_{e}^{2} d \mathrm{~V}$ ) of $I_{\alpha}=1.958 \times 10^{-7} \mathrm{erg} / \mathrm{cm}^{2}$-s (see MENoN 1962). Then the $12.8 \mu$ flux from $\mathrm{Ne}^{+}$can be estimated from $\mathrm{I}\left(\mathrm{Ne}^{+}\right)=\mathrm{I}_{\alpha}<\varepsilon\left(\mathrm{Ne}^{+}\right)>/<\varepsilon_{\alpha}>$. With $<\varepsilon\left(\mathrm{Ne}^{+}\right)>\mid<\varepsilon_{\alpha}>$ as computed in BGP, we obtain $\mathrm{I}\left(\mathrm{Ne}^{+}\right) \approx 7 \times 10^{-14} \mathrm{watt} / \mathrm{cm}^{2}$ for the expected flux from the Orion Nebula.

Other possible sources are the planetary nebulae. Taking $\varepsilon\left(\mathrm{Ne}^{+}\right)=2 \times 10^{--24} \mathrm{erg}-\mathrm{cm}^{3} / \mathrm{s}$ and using the data given by ALLER (1956) on the radius $r_{0}$,

\section{TABLE II}

EXPEOTED $12.8 \mu$ EMISSION FROM PLANETARY NEBULAE

\begin{tabular}{lccc}
\hline \hline NEBULA & $\left.\begin{array}{c}F_{\text {neb. }} \\
(\text { watt/cm }\end{array}{ }^{2}\right)$ & NEBULA & $\begin{array}{c}F_{\text {neb. }} \\
\left(\text { watt } / \mathrm{cm}^{2}\right)\end{array}$ \\
NGC 1535 & $2 \times \overline{10^{-16}}$ & NGC 6572 & $1 \times 10^{-15}$ \\
NGC 2149 & $9 \times 10^{-17}$ & NGC 6803 & $2 \times 10^{-17}$ \\
IC 418 & $2 \times 10^{-15}$ & NGC 6818 & $4 \times 10^{-16}$ \\
NGC 2392 & $3 \times 10^{-16}$ & NGC 6826 & $3 \times 10^{-16}$ \\
NGC 3242 & $1 \times 10^{-15}$ & NGC 7009 & $7 \times 10^{-16}$ \\
IC 4593 & $5 \times 10^{-17}$ & NGC 7027 & $2 \times 10^{-16}$ \\
NGC 6210 & $2 \times 10^{-16}$ & NGC 7662 & $7 \times 10^{-16}$ \\
NGC 6543 & $1 \times 10^{-15}$ & & \\
\hline \hline
\end{tabular}


distance $d$, and electron density $n_{e}$ for various planetary nebulae, the energy fluxes given in Table II were estimated from

$$
\mathrm{F}_{\text {neb. }}=\left\langle n_{e}^{2} \varepsilon\left(\mathrm{Ne}^{+}\right)>r_{0}^{3} / 3 d^{2} .\right.
$$

The best bets seem to be IC 418, NG(? $3242^{3}$ NGC 6543, and NGC 6572, from which fluxes $\sim 1^{-15} \mathrm{watt} / \mathrm{cm}^{2}$ are expected.

Nearby large galaxies may be detectable. If a source of luminosity (1) were at a distance of $1 \mathrm{Mpc}$ the flux would be $4 \times 10^{-15} \mathrm{watt} / \mathrm{cm}^{2}$; perhaps the $12.8 \mu$ flux from M 31 could be detected. The background flux from all galaxies out to a cosmological cut-off $R=5 \times 10^{27}$ seems to be quite high. Taking a mean $12.8 \mu$ luminosity per galaxy of $\left\langle\left(\mathrm{L}_{d}\left(\mathrm{Ne}^{+}\right)>\sim 4 \times 10^{42} \mathrm{erg} / \mathrm{s}\right.\right.$ and a mean number density of galaxies of

$$
n_{0} \sim 3 \times 10^{-75} \mathrm{~cm}^{-3}
$$

we calculate a cosmic background flux of

(4) $\mathrm{F}_{c}\left(\mathrm{Ne}^{+}\right) \sim<\mathrm{L}_{d}\left(\mathrm{Ne}^{+}\right)>n_{0} \mathrm{R} / 4 \pi$

$$
\sim 5 \times 10^{-13} \mathrm{watt} / \mathrm{cm}^{2}-\mathrm{sr} \text {. }
$$

Due to the differential red shift the line would be smeared into a continuum; the spectral shape of this continuum would depend on the large scale structure of the universe. Perhaps a measurement of this spectrum could shed some light on the controversial subject of cosmology.

Of the other infrared lines listed in Table I the most intense galactic lines are probably the lines from $\mathrm{C}^{+}$(which would also exist in $\mathrm{H} \mathrm{I}$ regions), $\mathrm{N}^{+}, \mathrm{N}^{+2}$, and $\mathrm{O}^{+2}$. However, these lie far into the infrared where detection is even more difficult; perhaps the lines from $\mathrm{Mg}^{+3}(4.49 \mu)$, $\mathrm{S}^{+3}(10.53 \mu), \mathrm{Ne}^{+4}(14.33)$, and $\mathrm{N}^{+2}$ (15.38) could be detected with less difficulty. While the mean galactic abundance of these ions is likely to be quite low, they may be abundant in certain high excitation planetary nebulae.

As yet, none of these lines have been detected unambiguously. However, a $12.8 \mu$ flux from $\mathrm{Ne}^{+}$may have been detected by WILDEY and MoRRay (1964) in their infrared observations of stellar radiation around $10 \mu$ (the observations actually included all radiation in the $8-14 \mu$ atmospheric window). Their preliminary observations indicated an infrared excess over the extrapolated black body spectra of about an order of magnitude for the two B stars $\alpha$ Leo and $\gamma$ Ori. As has been recently suggested ( $\mathrm{NeY}$ and GovLD, 1964), this excess radiation may be due to emission by $\mathrm{Ne}^{+}$in an ionized envelope of the star. A similar effect in the infrared spectra of cool stars would not be expected, since, even if the stars had gaseous envelopes, only a small amount of the gas could be ionized by the weak UV radiation from the star.

\section{II. $28 \mu$ Emission from Molecular Hydrogen.}

The possible existence of a $28 \mu$ emission line from interstellar hydrogen molecules has been known for a long time. The line results from the $\mathrm{J}=2$ to $\mathrm{J}=0$ quadrupole rotational transition in the (para) molecule. Here I shall consider two possible circumstances which might give rise to $28 \mu$ emission and shall make estimates of the associated fluxes.

\section{a) $28 \mu$ Emission During Protostar Collapse.}

It is often assumed that in the formation of stars out of condensations in the interstellar gas at some stage during the condensation the hydrogen gas has become molecular. The process of star formation out of molecular hydrogen gas has been treated recently (GouLD, 1964) and some of the results of this investigation seem to be relevant to infrared astronomy. When an initially cool molecular hydrogen gas sphere contracts and raises its temperature (collisional) excitation and emission of $28 \mu$ radiation increases and causes a rapid contraction. Depending slightly on the protostar mass, when the temperature of the contracting protostar has reached the relatively cool value $\sim 40-50 \mathrm{oK}$, the contraction approaches the freefall rate. The total galactic luminosity in $28 \mu$ radiation can be estimated from the rate of conversion of interstellar gas into stellar matter on the assumption that all star formation proceeds through a stage where the protostar is at least partly molecular. The resulting $28 \mu$ energy flux from star formation in the galactic disk is then about $3 \times 10^{-16} \mathrm{watt} / \mathrm{cm}^{2}$. This flux is quite small but may be detectable with some effort.

If star formation happens to be taking place abundantly in some nearby region, the intensity of $28 \mu$ radiation may be much larger. For example, the maximum $28 \mu$ luminosity of a protostar (which is proportional to the mass) of mass $100 \mathrm{M} \odot$ is about $100 \mathrm{~L} \odot$, and maintains this luminosity for $\sim 10^{6} \mathrm{yr}$. If such a source were at a distance of 500 pc (roughly the distance to the Orion Nebula), the flux received would be $10^{-13} \mathrm{watt} / \mathrm{cm}^{2}$, and this flux is quite large. Lines from other quadrupole rotational transitions should also be present, although their strength is likely to be smaller (by a factor $\lesssim 10$ ) ; their dura- 
tion of emission is also likely to be smaller. These lines are $J=3 \rightarrow 1(\lambda=17 \mu), J=4 \rightarrow 2(12 \mu)$, $\mathrm{J}=5 \rightarrow 3(9.4 \mu)$, etc.

b) $28 \mu$ Emission From Interstellar H I Clouds.

If the interstellar clouds are heated in inelastic cloud-cloud collisions (cf. GouLd, GoLd and SALPFTER, 1963), the principal cooling mechanism is by radiative de-excitation of the $\mathrm{J}=2$ state of $\mathrm{H}_{2}$ (and emission of $28 \mu$ radiation). The energy loss rate by this process per $\mathrm{cm}^{3}$ of intersteilar space would be

$$
d \mathrm{E}_{\mathrm{c}} / \mathrm{dt} \sim f m n^{2} \sigma v^{3}
$$

where $f\left(\sim \frac{1}{4}\right.$, a rough guess $)$ is the inelasticity, $m\left(\sim 6 \times 10^{24} \mathrm{gm}\right)$ the typical cloud mass, $\sigma\left(\sim 3 \times 10^{28} \mathrm{~cm}^{2}\right)$ the typical cloud cross section, and $v\left(\sim 10^{6} \mathrm{~cm} / \mathrm{s}\right)$ the mean cloud velocity ; one then obtains $d \mathrm{E}_{c} / d t \sim 5 \times 10^{-27} \mathrm{erg} / \mathrm{cm}^{8}-\mathrm{s}$. If this energy loss takes place throughout the galactic disk (volume: $\mathrm{V}_{d} \sim 2 \times 10^{66} \mathrm{~cm}^{3}$ ), the expected $28 \mu$ flux from the galactic disk would be $\left(d \mathrm{E}_{c} / d t\right) \mathrm{V}_{d} / 4 \pi r_{d}^{2} \sim 10^{-13}$ watt/om ${ }^{2}$, which is quite large. However, the inelasticity of cloud-cloud collisions may possibly be much smaller, and, of course, the "cloud model" for the interstellar medium is questionable.

This research has been supported in part by the National Science Foundation and in part by NASA through contract NsG-357.

\section{Manuscrit reçu le 14 septembre 1964.}

\section{BIBLIOGRAPHY}

AllmR L. H., 1956, Gaseous Nebulae (New York: John Wiley).

Burbidge G. R., Gould R. J. and Pottasoh S. R., 1963, Ap. J., 138, 945.

Gould R. J., 1963, Ap. J., 188, 1308 ; 1964, Ap. J., 140, 638.
Gould R. J., Gold T. and SALPETter E. E., 1963, $A p . J ., 138,408$.

Menon T. K., 1962, Ap.J., 136, 95.

Nex E. P. and Gould R. J.. 1964, Ap. J. 140, 388.

Wildey R. L. and MurRay B. C., 1964, Ap. J., 139, 435.

\section{Discussion}

D. W. Sorama. - It is wonderful that Dr. GouLd has discovered this line which stands out above the continuum and so may help to solve the cosmological problem. To illustrate the potential power of this method, I might just mention that the spectrum produced by differential red shift is $v^{8}$ for the steady state model and $\nu^{1 / 3}$ for the Einstein-de Sitter model. Fairly simple observations might thus suffice to distinguish between the two models.
L. Biermann. - From your figures, it seems that the emission of $\mathrm{Ne}^{+}$infrared lines must be a substantial part, say of the order of $10 \%$, of the luminosity of the central star of the H II regions. Is that so ?

R. Gould. - Yes, that is correct. The contribution of the $\mathrm{Ne}^{+}$line to the cooling of $\mathrm{H}$ II regions is treated in a paper by G. BuRBIDGE, S. РотTASOH and myself in $A p . J .138,945,(1965)$. 